\title{
CANALES ALTERNATIVOS DE ACCESO A LA PRENSA ESPAÑOLA EN INTERNET. LA NOTICIA FUERA DEL PERIÓDICO
}

\section{Begoña Ivars-Nicolás}

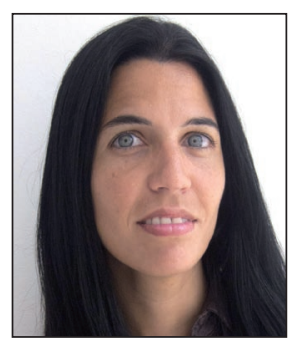

Begoña Ivars-Nicolás es profesora de periodismo en la Universidad Miguel Hernández (UMH) de Elche y de comunicación audiovisual en el Centro de Estudios Ciudad de la Luz adscrito a esta misma universidad. Doctora en antropología social, centra sus investigaciones en los medios de comunicación en la Red y en la repercusión de las TIC en la docencia. Coedita la revista digital de comunicación audiovisual Croma y codirige las ediciones del CICAP (Congreso Internacional de Comunicación Audiovisual y Publicidad de la UMH).

Universidad Miguel Hernández Av. de la Universidad, $s / n$ 03202 Elche (Alicante), España bivars@umh.es

\section{Resumen}

Se analizan los sistemas de información noticiosa a los que el lector accede y activa exclusivamente desde un diario digital, para recibir contenidos fuera del propio cibermedio. La escasa documentación y su constante evolución invitan a estudiar cómo las sindicaciones de contenidos o instrumentos similares se están integrando en los cibermedios. Se propone un modelo de análisis aplicado a siete periódicos nacionales españoles para conocer cuáles de estos servicios utilizan. Los diarios estudiados incorporan sistemas de difusión de la información basados en la interactividad y la personalización del lector: qué información desea recibir, cómo, cuándo y dónde. Destaca el papel del lector en el proceso de distribución mediante herramientas sociales.

\section{Palabras clave}

Acceso a la información, Búsqueda de información, Ciberperiodismo, Uso de la información.

Title: Alternative channels of access to the Spanish media on the internet. News without the newspaper

\begin{abstract}
We analyze the systems inside an online newspaper that allow access to news outside their own online media. It is important to know how mass media are using these tools because of the sparse documentation and its constant evolution. We propose a model of analysis applied to seven Spanish national newspapers to know how the information services, alternatives to navigation or search, are being used. Spanish newspapers distribution systems incorporate information based on interactivity and personalization: the kind of information you receive, how, when and where. We also emphasize the integration of social tools for sharing and disseminating news outside of the newspaper.
\end{abstract}

\section{Keywords}

Access to information, Research information, Cyberjournalism, Use of information.

Ivars-Nicolás, Begoña. "Canales alternativos de acceso a la prensa española en internet. La noticia fuera del periódico". El profesional de la información, 2012, julio-agosto, v. 21, n. 4, pp. 396-400.

http://dx.doi.org/10.3145/epi.2012.jul.11

\section{Introducción}

La consulta de contenidos periodísticos en internet se realiza principalmente mediante sistemas de recuperación de información (buscadores y hemerotecas digitales). Sobre este asunto han investigado algunos autores (Codina, 1995; Jiménez; González; Fuentes-Pujol, 2000; Abadal, 2002; López-Carreño, 2004; Guallar, 2007; Abadal; Guallar, 2008;
Guallar; Abadal, 2009a; Guallar; Rovira; Ruiz, 2010), que se han centrado en estudiar motores de búsqueda, sistemas de consulta o lenguajes de interrogación. Sin embargo, son más escasas las investigaciones sobre el acceso a contenidos mediante servicios alternativos al propio cibermedio (Centelles, 2005; Martínez-Rubio, 2008; Guallar; Abadal, 2009b; López-Aguirre, 2009; Abadal; Guallar, 2010; Rodríguez-Martínez; Codina; Pedraza-Jiménez, 2010). 
Miquel Centelles (2005) identifica tres modos de acceso a la información: la exploración, la recuperación y el filtraje. Guallar y Abadal (2009b) por su parte proponen cuatro tipos de sistemas de acceso a contenidos de prensa digital: acceso a cabeceras, búsqueda de información, seguimiento de información y agregación de contenidos (si bien estos últimos recopilan información de sistemas externos al ciberdiario).

Muchos diarios integran canales alternativos a su web para difundir sus contenidos. Estos son, según los autores que los han estudiado: alertas, sindicaciones, envío a email o dispositivos, registro de usuarios y personalización (RodríguezMartínez; Codina; Pedraza-Jiménez, 2010); sindicación, mapa del sitio, menús o índices temáticos (Martínez-Rubio, 2008); y alertas de noticias y envío de titulares (López-Aguirre, 2009).

Existe una tendencia creciente de uso de mecanismos alternativos para acceder a las noticias, abanderada por la evolución tecnológica y el desarrollo de la web social, que merecen un seguimiento.

\section{Objetivos y metodología}

Este texto estudia los sistemas de acceso a información noticiosa a los que el lector accede y activa exclusivamente desde el diario digital, para recibir los contenidos fuera del mismo. La investigación se centra en prensa generalista española en internet, excluyéndose instrumentos de navegación, buscadores, hemerotecas o archivos propios del cibermedio. También se omiten mecanismos externos que no implican un acceso previo, activación o selección del servicio por parte del lector en el cibermedio.

Primero se determinan cuáles son los servicios de acceso a la información existentes y se organizan según lo que quiere el usuario y cómo procede dentro del cibermedio para conseguirlo. Se parte de las propuestas de Centelles (2005) y Guallar y Abadal (2009b), situando los resultados en sus clasificaciones.

A partir de la bibliografía especializada se establecen criterios para comprobar la presencia y el funcionamiento básico de los servicios objeto de estudio y se organizan en una ficha de evaluación. Es un análisis cuantitativo porque determina qué servicios ofrece el periódico.

La muestra representativa de diarios se confecciona combinando datos del Estudio general de medios, OJD Interactiva y Alexa, disponibles en enero de 2012 (algunos sistemas no miden todos los medios e interesa conocer los datos referidos a sus webs). Se seleccionan las cuatro primeras cabeceras de cada lista resultando: El país, El mundo, $A B C, 20$ minutos, El periódico, Público y La vanguardia.

Partiendo de la home de cada cibermedio, se comprueba la existencia de cada servicio y se cumplimentan los criterios correspondientes. Se procede mediante la navegación y la observación del sitio exceptuando las páginas de pago. El estudio se realiza la primera semana de enero de 2012.

Este método permite valorar estos servicios en otros cibermedios especializados, de televisión o radio, por comunidades autónomas, e incluso extrapolarse a otros ámbitos.

\section{Resultados}

Los mecanismos de acceso que ofrecen los cibermedios estudiados se agrupan en cuatro categorías según lo que quiere el usuario y su procedimiento:

- Navegación: el lector quiere tener acceso a información y navega para localizarla mediante menús, índices, listas, titulares o mapa del sitio.

- Búsqueda: el lector quiere recuperar información y utiliza buscadores, hemerotecas o archivos.

- Suscripción/activación: el lector quiere recibir información y se suscribe en sistemas como alertas, envío de titulares y boletines, sindicación y descarga de widgets.

- Difusión: el lector quiere compartir información y la difunde en otras plataformas o dispositivos con opciones para compartir, en su red social, importa códigos, etc.

Esta propuesta parte de la realizada por Centelles (2005) pero, debido a la influencia de las plataformas sociales en la difusión de la información, se añaden los servicios para compartir información.

El texto se centra en los servicios englobados en las categorías de suscripción/activación y difusión. La ubicación de estos instrumentos en la propuesta de Guallar y Abadal (2009b) sería en las categorías de seguimiento de la información y la agregación de contenidos de actualidad, con la notable diferencia de que aquí se estudian sólo servicios que ofrece el cibermedio al usuario a través de su web para recibir los contenidos del medio en cuestión. En el análisis de Guallar y Abadal la recopilación de información se realiza a través de empresas o sistemas especializados y abarca contenidos de múltiples medios de comunicación.

Se establecen 6 tipos de servicios: alertas a teléfonos móviles, instrumentos para compartir, sindicación de contenidos, widgets, envío de titulares y envío de boletines

El uso de mecanismos alternativos que permiten el acceso a la información noticiosa, a los que el lector accede y activa exclusivamente desde un diario digital para recibir los contenidos fuera de este cibermedio, es generalizado y similar en la prensa generalista española. Se establecen seis servicios: alertas a teléfonos móviles, instrumentos para compartir, sindicación de contenidos, widgets, envío de titulares y envío de boletines.

Los datos muestran que el $71,4 \%$ de las cabeceras estudiadas proporciona acceso a servicios de alertas mediante mensajes sms o mms. Esta prestación, que es de pago y exige alta, se realiza a través de las principales compañías de telefonía móvil. El usuario elige la información y la frecuencia para recibirla. $A B C$ es el único diario que ofrece servicio de alertas gratuitas por email.

La sindicación de contenidos (RSS) aparece en todos los diarios. Se puede escoger el agregador o lector habitual y seleccionar la información (última hora, secciones, etc.). Se 


\begin{tabular}{|c|c|c|c|c|c|c|}
\hline El país & Público & El mundo & $A B C$ & 20 minutos & La vanguardia & El periódico \\
\hline \multicolumn{7}{|c|}{ Alertas móvil: pago; formato: sms/mms; personalización, frecuencia, etc. } \\
\hline Sí & & & Sí & Sí & Sí & Sí \\
\hline \multicolumn{7}{|c|}{ Agregación/lectores RSS: gratuito; formato: título, resumen y enlace noticia; personalización. } \\
\hline Sí & Sí & $\begin{array}{c}\text { Sí } \\
\text { Sindicación de } \\
\text { pago y gratuita }\end{array}$ & Sí & Sí & Sí & Sí \\
\hline \multicolumn{7}{|c|}{ Escritorios personales: gratuito; formato: título, resumen y enlace noticia; personalización. } \\
\hline $\begin{array}{l}\text { Convierte la } \\
\text { página de inicio } \\
\text { del navegador } \\
\text { mostrando la } \\
\text { portada del diario }\end{array}$ & & & & Sí & Sí & Sí \\
\hline \multicolumn{7}{|c|}{ Compartir (recomendar/añadir en redes y enviar email): gratuito; formato: enlace, tweet o titular. } \\
\hline $\begin{array}{c}\text { Sí } \\
\text { Ofrece código } \\
\text { para incluir } \\
\text { contenidos del } \\
\text { cibermedio en } \\
\text { blogs, webs } \\
\text { personales, etc. }\end{array}$ & $\begin{array}{c}\text { Sí } \\
\text { Recomendaciones } \\
\text { públicas en } \\
\text { Google }\end{array}$ & $\begin{array}{c}\text { Sí } \\
\text { Sistema de } \\
\text { suscripción que } \\
\text { permite seguir } \\
\text { y que te sigan } \\
\text { otros lectores }\end{array}$ & $\begin{array}{c}\text { Sí } \\
\text { Recomendaciones } \\
\text { públicas en } \\
\text { Google }\end{array}$ & Sí & $\begin{array}{c}\text { Sí } \\
\text { Recomendaciones } \\
\text { públicas en } \\
\text { Google }\end{array}$ & Sí \\
\hline \multicolumn{7}{|c|}{ Titulares: gratuito; formato: título, resumen y enlace noticia; personalización. } \\
\hline Sí & & & $\begin{array}{c}\text { Sí } \\
\text { Servicio gratis de } \\
\text { alertas por email }\end{array}$ & & Sí & Sí \\
\hline \multicolumn{7}{|c|}{ Boletines: gratuito; formato: título, resumen y enlace noticia; personalización. } \\
\hline & & & Sí & Sí & Sí & \\
\hline
\end{tabular}

Tabla 1. Organización de las cabeceras por servicio y características

suele visualizar el título, resumen y enlace a la noticia en el cibermedio, dependiendo de la cabecera. El mundo proporciona una versión de pago y otra gratuita.

El periódico, La vanguardia y 20 minutos $(42,8 \%)$ ofrecen también sindicaciones a través de widgets. Estas aplicaciones se instalan en el escritorio del ordenador o dispositivo similar generando una versión reducida y personalizada del diario (titulares por secciones que enlazan con la noticia) que se actualiza por sí misma. 20 minutos ofrece Mini20 que permite activar alertas de otros medios de comunicación, y El país ofrece convertir la página de inicio del navegador mostrando la portada del diario.

Los 7 periódicos ofrecen instrumentos para compartir contenidos o difundirlos en plataformas sociales. En el nodo en el que se desarrolla la noticia se ofrecen servicios para que el usuario la comparta o la recomiende añadiéndola (enlace o titular vinculado al medio) a plataformas sociales. También se pueden enviar noticias por correo electrónico (100\%) y hacer recomendaciones públicas en Google (42,8\%). El país ofrece código para incluir enlaces del cibermedio en webs personales, blogs o en la home de Google. Las opciones para seguir al medio en redes sociales no se contemplan en este análisis porque son accesibles sin necesidad de entrar en el cibermedio.

Casi todos los diarios tienen mecanismos para enviar titulares $(57,1 \%)$ o boletines $(28,6 \%)$ por email. El usuario se registra y selecciona el tipo de información, secciones y la edición. Son recopilaciones de titulares con resumen y enlace al cibermedio. En los boletines, los titulares se complementan con imágenes y listados de otras secciones o servicios.

En cuanto a cantidad, Público y El mundo ofrecen menos servicios de envío 
de información. Se apoyan en sistemas de RSS para proporcionar la información que el resto de diarios ofrece mediante alertas, widgets o envío de titulares.

Todos los mecanismos ofrecen información de actualidad del medio en concreto y se activan registrándose o introduciendo una dirección de email o número móvil donde recibir la información, siempre enlazada con el cibermedio. Estos sistemas de valor añadido suelen ubicarse en la página de inicio. El área de registro o suscripción y los enlaces a las sindicaciones de contenidos suelen encontrarse en la parte superior de la página, coincidiendo con los vínculos al buscador y a la hemeroteca. Otros mecanismos como alertas, envío de titulares o de boletines suelen integrarse en una sección con servicios varios en el menú principal o al final de la página. En todas las cabeceras el usuario puede compartir, añadir, recomendar o comentar noticias en redes sociales o enviarlas a un amigo por email.

\section{Discusión}

La evolución de los sistemas de acceso y recuperación de la información se ha desarrollado en 3 fases:

1) Inicialmente se navegaba por el cibermedio para localizar la información mediante menús. Esta navegación se implementó con estructuras como índices, titulares o mapas contextuales para facilitar y agilizar el acceso a los contenidos.

\section{Algunos medios emplean sólo sistemas} de RSS para proporcionar la información que el resto de diarios ofrece mediante alertas, widgets o envío de titulares

2) Posteriormente, la información se ha organizado en bases de datos conformando la memoria del sitio (Canavilhas, 2007; Abadal; Guallar, 2010). Estos sistemas de almacenamiento permiten recuperar contenidos usando sistemas de consulta como motores de búsqueda y hemerotecas.

3) A estos servicios de valor añadido se suman otros alternativos de acceso a la información:

- el lector define sus preferencias en el diario y, cuando éstas se cumplen el medio le proporciona la información solicitada, personalizada y en un dispositivo externo al cibermedio (en ocasiones se trata de una recuperación asíncrona);

- instrumentos para compartir información noticiosa, es decir, el cibermedio comunica a los lectores y estos lo transmiten a otros usuarios (a nivel individual o mediante plataformas sociales produciéndose un efecto multiplicador);
- aplicaciones para instalar versiones reducidas, personalizadas y actualizables del diario.

Los instrumentos son: alertas a teléfonos móviles, instrumentos para compartir, sindicación de contenidos, widgets, envío de titulares y envío de boletines. La información recibida suele ser parcial y se compone del titular de la noticia, un resumen y el enlace al medio. La apariencia de los datos es similar para todos los servicios, la diferencia es el mecanismo por el cual se recibe la información: escritorio personalizado, buscador o navegador, email, etc.

Por ello, un cibermedio debe ofrecer al menos dos tipos de servicios: un sistema de envío de información personalizada (sindicación, alertas, titulares o boletines); y un instrumento para compartir información en plataformas sociales.

Las cabeceras españolas disponen de sindicación de contenidos y servicios para compartir información, situándose en la tercera fase de desarrollo antes expuesta. Más de la mitad de los diarios complementan estos mecanismos con la posibilidad de enviar por correo electrónico recopilaciones de titulares o boletines a los lectores, incluso diarios de escritorio personalizados. La noticia se puede recibir mediante un sms, en forma de email, al encender el ordenador o al abrir el navegador.

Es notable la inclinación a centrarse en el usuario y sus necesidades. Si esta tendencia crece, estos sistemas pueden ser relevantes en el modelo de negocio del cibermedio. Para ello la calidad del servicio es el mayor reclamo, es decir, obtener un documento organizado, contextualizado, adapta- 
do, intuitivo e interactivo por el que el lector esté dispuesto a pagar.

Los avances tecnológicos, la evolución de la audiencia y su rol en internet en menos de dos décadas derivan en un lector-usuario a tres velocidades:

- el que se limita a leer la información noticiosa navegando por el sitio web;

- el que navega, interactúa y utiliza los instrumentos del cibermedio;

- el usuario personalizado, actualizado y social, que recibe la información de actualidad a través de sistemas de envío de noticias o plataformas sociales. Este usuario accede poco al diario (sólo para ampliar la información o suscribirse a servicios de información personalizada). Además, ha adquirido un papel relevante en el proceso de distribución, difundiendo los contenidos, lo que exige adaptaciones tecnológicas por el medio.

La Red, como medio de comunicación emergente, ha obligado a idear estructuras de comunicación e información alterando los procesos de trabajo habituales en el periodismo, por ejemplo, en el acceso a la información y, por consiguiente, en su distribución. Los cibermedios de prensa españoles se encuentran en una fase de innovación en cuanto a estrategias y herramientas de difusión de la información noticiosa. No sólo están adaptándose a las características inherentes de internet y beneficiándose de ellas, sino que están integrando formas de comunicación social aprovechando el comportamiento de los usuarios en plataformas externas como redes sociales o blogs.

Estos escenarios contribuyen a la difusión de los contenidos periodísticos trasladando la noticia del diario a un espacio que se sitúa más allá del propio cibermedio y que cada usuario comparte con su comunidad.

\section{Bibliografía}

Abadal, Ernest. "Elementos para la evaluación de interfaces de consulta de bases de datos". El profesional de la información, 2002, sept.-oct., v. 11, n. 5, pp. 349-360.

http://www.elprofesionaldelainformacion.com/conteni dos/2002/septiembre/3.pdf

Abadal, Ernest; Guallar, Javier. "Les hemeroteques digitals de la premsa catalana: anàlisi dels diaris catalans de més difusió". En: $11^{\text {es }}$ Jornades catalanes de documentació, 2008, pp. 149-162. Barcelona: Cobdc.

http://eprints.rclis.org/archive/00013686/01/jornades2008 _guallar-abadal_hemeroteques_diaris.pdf

Abadal, Ernest; Guallar, Javier. Prensa digital y bibliotecas. Gijón: Trea, 2010, 176 p. ISBN: 978849704462

Centelles, Miquel. “Taxonomía para la categorización y la organización de la información en sitios web". Hipertext.net, 2005. http://www.hipertext.net/web/pag264.htm

Canavilhas, João. Webnoticia. Propuesta de modelo periodístico para la www. Covilhà: Livros Labcom, 2007. ISBN: 9789728790912

http://www.livroslabcom.ubi.pt/pdfs/20110823-canavil has_webnoticia_final.pdf

Codina, Lluís. "Recuperación de la información e hipertex- tos: sus bases lógicas y su aplicación a la documentación periodística". En: Fuentes-Pujol, Maria-Eulàlia (edit.). Manual de documentación periodística. Madrid: Síntesis, 1995, pp. 213-230. ISBN: 9788477383048

Guallar, Javier. "La renovación de los diarios digitales: rediseños y web 2.0". El profesional de la información, 2007, v. 16 , n. 3, pp. 235-242.

http://www.elprofesionaldelainformacion.com/contenidos/ 2007/mayo/08.pdf

http://dx.doi.org/10.3145/epi.2007.may.08

Guallar, Javier; Abadal, Ernest. "Evaluación de hemerotecas de prensa digital: indicadores y ejemplos de buenas prácticas". El profesional de la información, 2009a, mayo-junio, v. 18, n. 3, pp. 255-269.

http://eprints.rclis.org/handle/10760/13048\#.T-bhNit1DEU http://dx.doi.org/10.3

145/epi.2009.may.02

Guallar, Javier; Abadal, Ernest. "Fuentes de información sobre prensa digital: una propuesta de clasificación". En: I Congreso internacional de ciberperiodismo y web 2.0, 2009b, pp. 11-13, Bilbao.

http://eprints.rclis.org/17176

Guallar, Javier; Rovira, Cristòfol; Ruiz, Sara. "Multimedialidad en la prensa digital. Elementos multimedia y sistemas de recuperación en los principales diarios digitales españoles". El profesional de la información, 2010, nov.-dic., v. 19, n. 6, pp. 620-629.

http://eprints.rclis.org/handle/10760/15088\#.T-bphSt1DEU http://dx.doi.org/10.3145/epi.2010.nov.08

Jiménez, Àngels; González, Alfons; Fuentes-Pujol, MariaEulàlia. "Las hemerotecas digitales de la prensa en internet". El profesional de la información, 2000, mayo, v. 9, n. 5, pp. 15-22.

http://www.elprofesionaldelainformacion.com/contenidos/ 2000/mayo/2.pdf

López-Aguirre, José-Luis. "Estudio de servicios y productos ciberdocumentales en la prensa digital mexicana". Documentación de las ciencias de la información, 2009, v. 32, pp. 93-132.

http://revistas.ucm.es/inf/02104210/articulos/DCIN090911 0093A.PDF

López-Carreño, Rosana. "Análisis taxonómico de los portales periodísticos españoles". Anales de documentación, 2004, n. 7, pp. 123-140.

http://digitum.um.es/xmlui/handle/10201/3906

Martínez-Rubio, Rosa. "La recuperación de la información en los periódicos digitales valencianos". En: López-García, Guillermo. Comunicación local y nuevos formatos periodísticos en internet: cibermedios, confidenciales y weblogs. Valencia: Servei de Publicaciones de la Universitat de València, 2008, pp. 99-140.

http://www.cibermediosvalencianos.es/comloc/Martinez.pdf Rodríguez-Martínez, Ruth; Codina, Lluís; Pedraza-Jiménez, Rafael. "Cibermedios y web 2.0: modelo de análisis y resultados de aplicación". El profesional de la información, 2010, enero-febrero, v. 19, n. 1, pp. 35-44.

http://repositori.upf.edu/bitstream/handle/10230/13140/ Pedraza_epi_1.pdf?sequence $=1$

http://dx.doi.org/10.3145/epi.2010.ene.05 